\title{
Photonic sampling of broadband QAM microwave signals exploiting interleaved optical Nyquist pulses
}

\author{
Valeria Vercesi $^{1}$, Daniel Onori ${ }^{2}$, John Davies ${ }^{3}$, Alwyn Seeds ${ }^{1}$ and Chin-Pang Liu ${ }^{1}$ \\ ${ }^{1}$ Department of Electronic \& Electrical Engineering, University College London, Torrington Place, London WC1E 7JE, United Kingdom \\ ${ }^{2}$ TeCip Institute, ScuolaSuperiore Sant'Anna, Via Giuseppe Moruzzi, 1, 56127 Pisa, Italy \\ ${ }^{3}$ THALES UK, 350 Longwater Avenue, Green Park, Reading, RG2 6GF, United Kingdom \\ v.vercesi@ucl.ac.uk
}

\begin{abstract}
We performed photonic sampling of 6 Gbaud 16-QAM signals at $20 \mathrm{GHz}$ sampling frequency by interleaving and time-aligning two $10 \mathrm{GHz}$ Nyquist optical sampling pulse trains using a SMF as the dispersive medium.

OCIS codes: (000.0000) General; (000.0000) General [8-pt. type] For codes, see http://www.osapublishing.org/submit/ocis/
\end{abstract}

\section{Introduction}

Photonics has been investigated and employed to increase the performance of electronic analog to digital converters (ADCs) for a long time now, to the extent that the first fully photonics-based radar system has already been realized [1]. Many demonstrated photonic ADC systems have employed mode-locked lasers as the sampling pulse sources [1-2]. However, such pulsed optical sources require constant stability adjustments and do not allow flexible sampling frequencies [3]. Our aim is to build a fully tunable photonic based ADC architecture suitable for the reception of ultra-wideband microwave signals with multilevel vector modulation formats. To this end, we propose a flexible cavity-less optical Nyquist pulse source employed in a system that can be easily reconfigurable and scalable to achieve higher sampling frequencies while employing lower frequency electronic ADCs. Although the recently proposed all-optical Nyquist pulse generation techniques [4] are applicable to the sampling of microwave frequency signals, their implementations can be complicated as they require careful electrical/optical path matching, frequency multiplication, and/or more than one modulator. Therefore, we generated optical Nyquist pulses using an arbitrary waveform generator (AWG) followed by a Mach Zehnder modulator (MZM), implementing a simpler, highly stable and flexible technique, allowing for precise control of the repetition rate and pulse shape as well as the pulse amplitude. When more than one wavelength is used, this technique can simultaneously generate multiple Nyquist pulse trains with the same repetition rate but with different wavelengths. Using a few kilometers of standard single mode fiber (SMF) as a dispersive medium, the generated optical Nyquist pulse trains become uniformly interleaved in time, decreasing the pulse interval and, effectively, increasing the sampling frequency. The proposed direct sampling of microwave frequency signals with Nyquist pulses is expected to find applications in wireless communications, antenna remoting and radar systems [1-4]. In this work, we demonstrate the sampling and successful demodulation of 6 Gbaud quadrature amplitude modulated (QAM) signals using two interleaved $10 \mathrm{GHz}$ optical Nyquist pulse trains to perform the direct photonic sampling of the incoming signal at an overall sampling frequency of $20 \mathrm{GHz}$. We achieved low error vector magnitude (EVM) values of less than $10 \%$ up to a carrier frequency of $15 \mathrm{GHz}$ considering signal bandwidths up to $8.1 \mathrm{GHz}$.

\section{Generation of optical Nyquist pulses for photonic sampling}

We generated optical periodic Nyquist pulses, suitable for flexible photonic sampling operations, starting from electronically synthesized periodic Nyquist pulses. To this end, we calculated the pulse waveform files to be loaded to the AWG to generate the electrical signals to drive the MZM, which in turn produced the optical pulses for the photonic sampling process. A single, positive Nyquist pulse is generally described in the time-domain by:

$$
h(t)=\frac{\sin \left(\frac{\pi t}{T_{S}}\right)}{\left(\frac{\pi t}{T_{S}}\right)} \frac{\cos \left(\frac{\pi \beta t}{T_{S}}\right)}{\left(1-\left(\frac{2 \beta t}{T_{S}}\right)^{2}\right)}
$$

where $T_{S}$ is the pulse duration between zero-crossings and $\beta$ is the roll-off factor. A periodic pulse train made up of several Nyquist pulses spaced by 100 ps can be obtained by summing a series of uniformly time-shifted individual Nyquist pulses, each properly truncated in time. Considering the $25 \mathrm{GHz}$ bandwidth of the AWG, we generated pulses with $\beta=0.5$ and $T_{S}=25 p s$. The FFT of a single Nyquist pulse generated with these parameters has its maximum frequency component at $30 \mathrm{GHz}$ while the FFT of a $10 \mathrm{GHz}$ pulse train has its maximum component at 
$20 \mathrm{GHz}$ that is within the AWG bandwidth (Fig. 1a). Individual pulses are orthogonal to each other in the timedomain as the time interval of the periodic pulse train (100 ps) is a multiple of the time duration between zerocrossings ( $25 \mathrm{ps}$ ), and therefore individual pulse peaks only occur at the zero-crossing points of other pulses (Fig. 1b). Optical Nyquist pulses were obtained by pulse carving with an MZM driven by the AWG. To preserve orthogonality in the carved optical pulses and meet the Nyquist ISI-free criterion, the DC bias voltage for the MZM was set so that the zero-crossing points of the AWG generated Nyquist pulse trains were at the half-wave voltage of the MZM, $V_{\pi}$, at which the output optical power should ideally be zero. The negative parts of the electrical pulse trains were "flipped" upwards about the zero-voltage level. The pulse carving process is illustrated in Fig. 1c. The concave-upward transfer characteristic of the MZM around $\mathrm{V}_{\pi}$ produces a reduction in the FWHM of the carved pulses. The amount of pulse compression is a function of the peak voltage of the input electrical Nyquist pulses to the MZM. For example, if the peak voltage is set to $0.5 \mathrm{~V}_{\pi}$ a pulse width compression of about $22 \%$ is obtained.
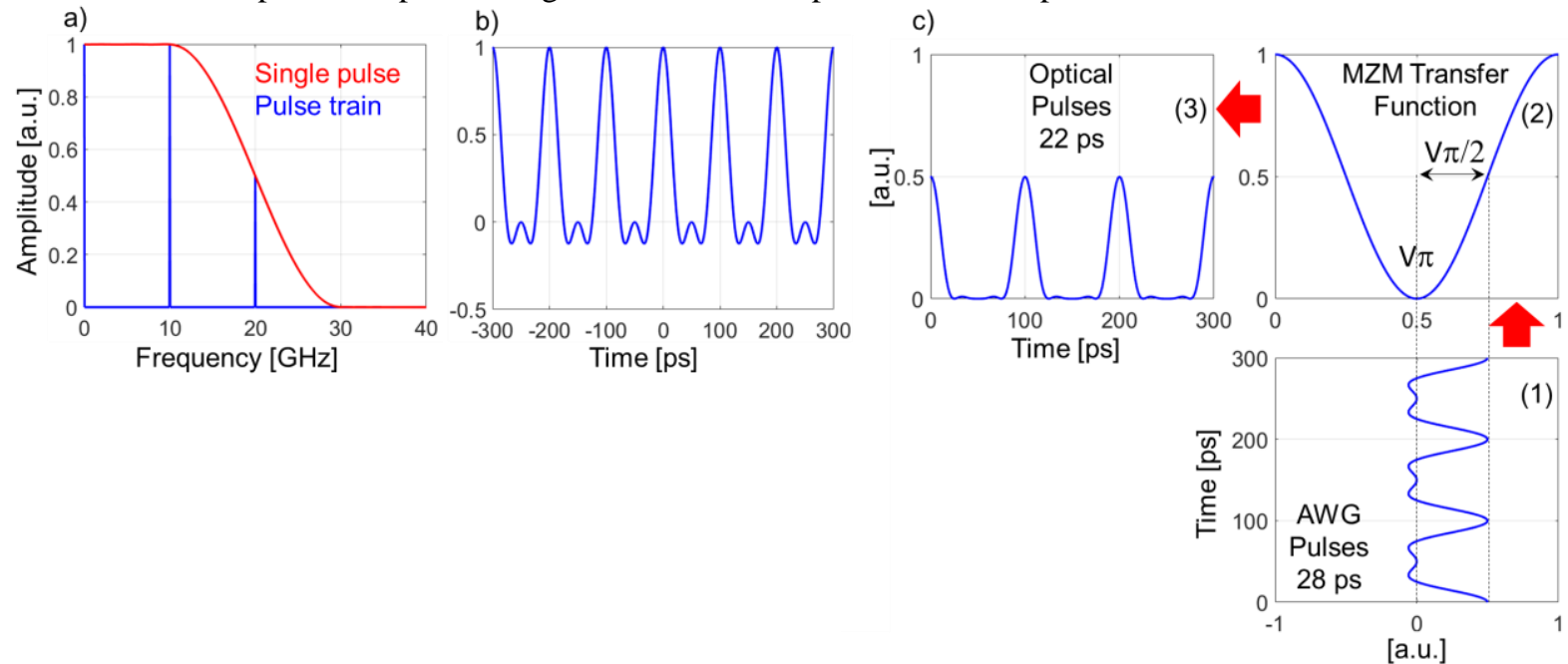

Fig. 1. Numerical calculations of:. a) frequency spectra of one single Nyquist pulse (red) and the corresponding $10 \mathrm{GHz}$ periodic pulse train (blue) with $\beta=0.5$ and $\mathrm{Ts}=25 \mathrm{ps}$; b) time-domain waveform of the $10 \mathrm{GHz}$ Nyquist periodic pulse train. $\mathrm{c}$ ) optical Nyquist pulse generation by pulse carving with a MZM driven with the electrical Nyquist pulses from the AWG: (1) electrical Nyquist pulses generated by the AWG, pulse width $=28 \mathrm{ps}$; (2) MZM transfer function and (3) $10 \mathrm{GHz}$ carved optical Nyquist pulses from (1), pulse width $=22 \mathrm{ps}$.

\section{Photonic sampling experiment}

The implemented architecture is shown in Fig. 2. Two CW laser sources (LASER1,2) were combined and sent to a first $40 \mathrm{GHz}$ Lithium Niobate intensity modulator (MZM1) to obtain two trains of optical Nyquist pulses according to the technique described above. Using a $25 \mathrm{GHz}$ Keysight M8195A AWG followed by MZM1, $10 \mathrm{GHz}$ optical Nyquist pulses with a peak pulse power of $32 \mathrm{~mW}$ and a FWHM of around 22 ps were generated on both CWs. The two optical Nyquist pulse trains at the output of MZM1 were superimposed in the time domain. Afterwards, a span of SMF was employed as a dispersive medium to time align the two $10 \mathrm{GHz}$ pulse trains to obtain equally spaced pulses with a higher repetition rate of $20 \mathrm{GHz}$. Indeed, the pulse trains were sent through $2 \mathrm{~km}$ of SMF where time alignment occurs thanks to the about $18 \mathrm{ps} / \mathrm{nm} / \mathrm{km}$ dispersion, according to the equation $\Delta \tau=$ $\Delta \lambda \times \mathrm{D} \times \mathrm{L}$, where $\Delta \tau$ is the required time shift, i.e., $50 \mathrm{ps}(1 / 20 \mathrm{GHz}), \Delta \lambda(1.25 \mathrm{~nm})$ is the spacing of the two CWs, D is the fiber dispersion and finally $\mathrm{L}$ the fiber length. A fine tuning of $\Delta \tau$ can be obtained by adjusting the CWs' spacing. When MZM2 was driven by the input signal, photonic sampling was thus performed by the $20 \mathrm{GHz}$ optical pulse trains going through MZM2. Finally, the two pulse trains were separated by optical filtering and processed individually to reconstruct the incoming signal. At this stage, the digitization process was performed with a real-time oscilloscope. So, the photodetected Nyquist pulses were captured using two channels of a $36 \mathrm{GHz}$ realtime LeCroy oscilloscope working at $80 \mathrm{GSa} / \mathrm{s}$, which is a necessary condition to use the full $36 \mathrm{GHz}$ input electrical bandwidth. The oscilloscope was synchronized with the AWG so that the received Nyquist pulse peaks were captured precisely by the oscilloscope at one out of every eight sampling instances. The future approach will be to employ two $10 \mathrm{GSa} / \mathrm{s}$ digitizers, synchronized with the Nyquist pulse peaks, without any anti-aliasing filter at the input. Digital signal processing (DSP) in LabVIEW was implemented to decimate the two captured channel waveforms from $80 \mathrm{GSa} / \mathrm{s}$ to $10 \mathrm{GSa} / \mathrm{s}$, retaining only those samples holding the amplitude values of the pulse peaks, and to interleave the two streams of samples obtained from the received signals.

Photonic sampling and demodulation of vector modulated microwave signals was performed. Here we report measurements of 6 Gbaud 16-QAM signals with the carrier frequencies centered at $5 \mathrm{GHz}$ and $15 \mathrm{GHz}$, generated 
using the AWG and input to MZM2. The root raised cosine function with a roll-off factor of 0.35 was used to shape the QAM signals, leading to a modulation bandwidth of $8.1 \mathrm{GHz}$. Demodulation and analysis of the sampled QAM signals was performed in LabVIEW with the same root raised cosine pulse-shaping filter. The left side of Fig. 3 shows the measured constellation diagrams where the EVMs were about $6.3 \%$, and $9.7 \%$ at $5 \mathrm{GHz}$ and $15 \mathrm{GHz}$, respectively. The right side of Fig. 3 shows the corresponding spectra of the reconstructed signals in the first Nyquist zone $(0-10 \mathrm{GHz})$. This result demonstrates the capability of the proposed photonic sampling technique to sample and process ultra-wideband microwave signals with multilevel vector modulation formats.

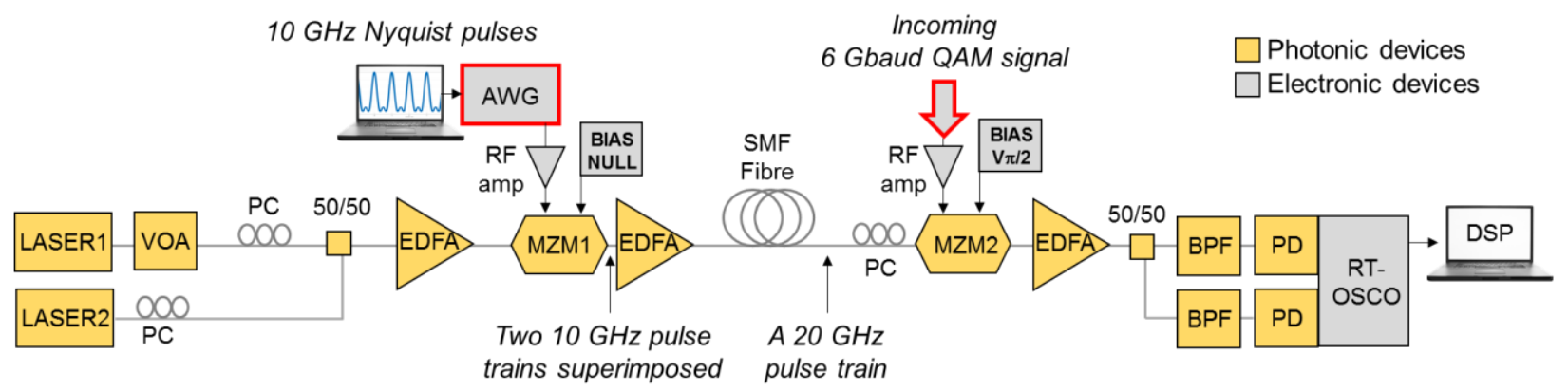

Fig. 2. Setup for the photonic sampling experiment: VOA: variable optical attenuator; EDFA: erbium doped fiber amplifier; MZM: Mach Zehnder modulator; BPF: bandpass filter; PD: photodiode; RT-OSCO: real time oscilloscope and DSP: digital signal processing.
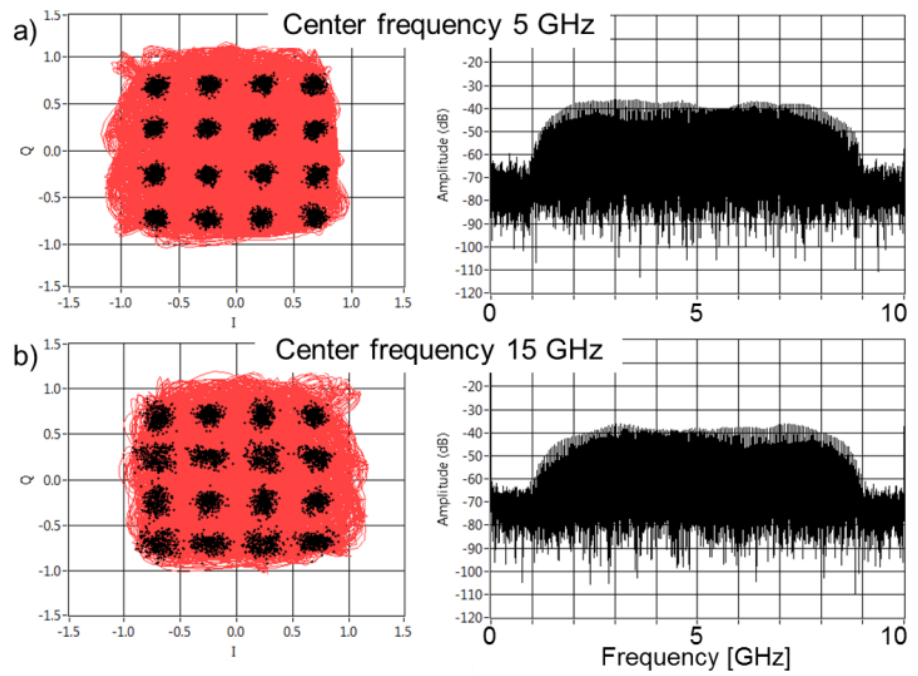

Fig. 3. Constellation diagram and frequency spectra of the received 6 Gbaud 16 QAM signal centered at a) $5 \mathrm{GHz}$, EVM $=6.3 \%$, and b) $15 \mathrm{GHz}$, $\mathrm{EVM}=9.7 \%$.

\section{Conclusions}

A $20 \mathrm{GHz}$ photonic sampling technique based on the use of two interleaved $10 \mathrm{GHz}$ optical Nyquist pulses has been investigated, demonstrating the sampling and successful demodulation of 6 Gbaud 16-QAM signals centered at both $5 \mathrm{GHz}$ and $15 \mathrm{GHz}$. The use of one SMF to time align the two optical pulse trains further allows for a flexible sampling frequency because both the pulse repetition rate as well as the wavelength spacing are easily adjusted. Future activities will include the optimization of the system, e.g., MZM nonlinearities compensation, and then measurement of the achievable effective number of bits.

\section{Acknowledgements}

This work has been funded by the United Kingdom Engineering and Physical Sciences Research Council (EPSRC) (standard grant no.: EP/M021939/1)

\section{References}

[1] P. Ghelfi, F. Laghezza, F. Scotti, G. Serafino, A. Capria, S. Pinna, D. Onori, C. Porzi, M. Scaffardi, A. Malacarne, V. Vercesi, E. Lazzeri, F.

Berizzi and A. Bogoni, "A fully photonics-based coherent radar system," Nature 507, 341-345 (2014).

[2] G. C. Valley, "Photonic analog-to-digital converters," Opt. Express 15(5), 1955-1982 (2007).

[3] A. O. J. Wiberg, L. Liu, Z. Tong, E. Myslivets, V. Ataie, B. P.-P. Kuo, N. Alic, and S. Radic "Photonic preprocessor for analog-to-digitalconverter using a cavity-less pulse source", Opt Express 20, b419-b427 (2012)

[4] C.S. Bres, "Advances in frequency comb synthesis-based Nyquist pulse-train carver and application in optical system", ICTON, Th.B1.1 (2015). 